\title{
Narrowband frequency-drift structures in solar type IV bursts
}

\author{
Yukio Nishimura ${ }^{1}$, Takayuki Ono ${ }^{1}$, Fuminori Tsuchiya ${ }^{2}$, Hiroaki Misawa ${ }^{2}$, Atsushi Kumamoto ${ }^{1}$, \\ Yuto Katoh ${ }^{1}$, Satoshi Masuda ${ }^{3}$, and Yoshizumi Miyoshi ${ }^{3}$ \\ ${ }^{1}$ Space and Terrestrial Plasma Physics Laboratory, Department of Geophysics, Graduate School of Science, \\ Tohoku University, Sendai, Miyagi 980-8578, Japan \\ ${ }^{2}$ Planetary Plasma and Atmospheric Research Center, Graduate School of Science, Tohoku University, Sendai, Miyagi 980-8578, Japan \\ ${ }^{3}$ Solar-Terrestrial Environment Laboratory, Nagoya University, Nagoya, Aichi 464-8601, Japan
}

(Received February 18, 2013; Revised August 21, 2013; Accepted September 13, 2013; Online published December 6, 2013)

\begin{abstract}
We have established the Zao Solar Radiospectrograph (ZSR), a new solar radio observation system, at the Zao observatory of Tohoku University, Japan. We observed narrowband fine structures with type IV bursts with ZSR on 2 and 3 November 2008. The observed fine structures are similar to fiber bursts in terms of the drift rates and the existence of emission and absorption stripes. Statistical analysis of the drift rates, however, shows that the observed fine structures are different from the ordinary fiber bursts as regards the sense and the magnitude of their drift rates. First, the observed drift rates include both positive and negative rates, whereas ordinary fiber bursts are usually characterized by negative drift rates. Second, the absolute values of the observed drift rates are tens of $\mathrm{MHz} \mathrm{s}^{-1}$, whereas the typical drift rate of fiber bursts at $325 \mathrm{MHz}$ is approximately $-9 \mathrm{MHz} \mathrm{s}^{-1}$. In addition, all fine structures analyzed have narrow emission bands of less than $17 \mathrm{MHz}$. We also show that the observed narrowband emission features with drift rates of approximately $40 \mathrm{MHz} \mathrm{s}^{-1}$ can be interpreted as the propagation of whistler-mode waves, which is the same process as that underlying fiber bursts.
\end{abstract}

Key words: Sun, radio, type IV burst, fiber burst, fine structure.

\section{Introduction}

Solar type IV bursts are intense radio phenomena that follow solar flares. They have wideband continuous spectra that extend from meter into decimeter wavelengths (e.g., Wild et al., 1963). Following their discovery by Elgarøy (1959), many observations of type IV bursts have revealed that they are accompanied by several kinds of fine structure. One of the most prominent fine-structure in type IV bursts are fiber bursts. They were first detected by Young et al. (1961). They are usually referred to as 'intermediate drift bursts' (IMDs). Detection of the same kind of burst has also been reported by Thompson and Maxwell (1962), Slottje (1972), Aurass et al. (1987), and many others. Recently, observations in the decimeter and microwave range have been made by many authors. For example, Benz and Mann (1998) investigated fiber bursts in the frequency range of 1-3 GHz. Chernov et al. (2001) and Fernandes et al. (2003) reported observational results with high resolution at frequencies above $1 \mathrm{GHz}$. The main characteristics of fiber bursts are as follows: (i) their drift rate is usually negative, (ii) the $\mathrm{drift} /$ frequency ratio, $|(\mathrm{d} f / \mathrm{d} t) / f|$, is on the order of $0.04-0.1 \mathrm{~s}^{-1}$, (iii) their instantaneous bandwidth is approximately $2 \%$ of the emission frequency (Benz and Mann, 1998), and (iv) they are accompanied a paralleldrift absorption band in the background continuum radiation (e.g., Chernov, 2006). Kuijpers (1975) proposed an

Copyright (c) The Society of Geomagnetism and Earth, Planetary and Space Sciences (SGEPSS); The Seismological Society of Japan; The Volcanological Society of Japan; The Geodetic Society of Japan; The Japanese Society for Planetary Sciences; TERRAPUB.

doi:10.5047/eps.2013.09.009 emission mechanism for fiber bursts consisting of the interaction of a Langmuir and a whistler-mode wave. This produces a left-handed polarized ordinary (L-O) mode wave that can escape from the emission region. According to this theory, the drift rate of the emission frequency is interpreted by the group velocity of the whistler-mode waves. The proposed mechanism is generally accepted, although it has also attracted some criticism (e.g., Bernold and Treumann, 1983). Recently, Aurass et al. (2005) showed the validity of this process for estimating magnetic-field strength with fiber bursts. Previous studies have also proposed another emission mechanism which involves Alfvén velocity as the speed of the motion of emission source (e.g., Treumann et al., 1990; Kuznetsov, 2006; Karlický et al., 2013). Observations and the theoretical background of fiber bursts are reviewed by Chernov (2006).

Many radio spectrometers for observations of solar radio bursts have been operated in the meter-wave range, such as Phoenix in Switzerland (Benz et al., 1991), Brazilian Solar Spectroscope (BSS) in Brazil (Sawant et al., 2001), Hiraiso Radio Spectrograph (HiRAS) in Japan (Kondo et al., 1995), and Culgoora in Australia (Prestage et al., 1994). Since the resolution of these wide-band spectrometers is often limited to a time resolution of $0.1 \mathrm{~s}$ and a frequency resolution of $1 \mathrm{MHz}$, it is not sufficient to investigate the fine-structure details of radio bursts such as fiber bursts, which have approximately $1 \mathrm{MHz}$ of instantaneous bandwidth at a frequency near $300 \mathrm{MHz}$. There are radiospectrographs with higher time resolution of milliseconds (e.g., Fu et al., 1995; Jiřička and Karlický, 2008; Dạbrowski et al., 2011), which have revealed a new class of fine structures of radio bursts 
(a)

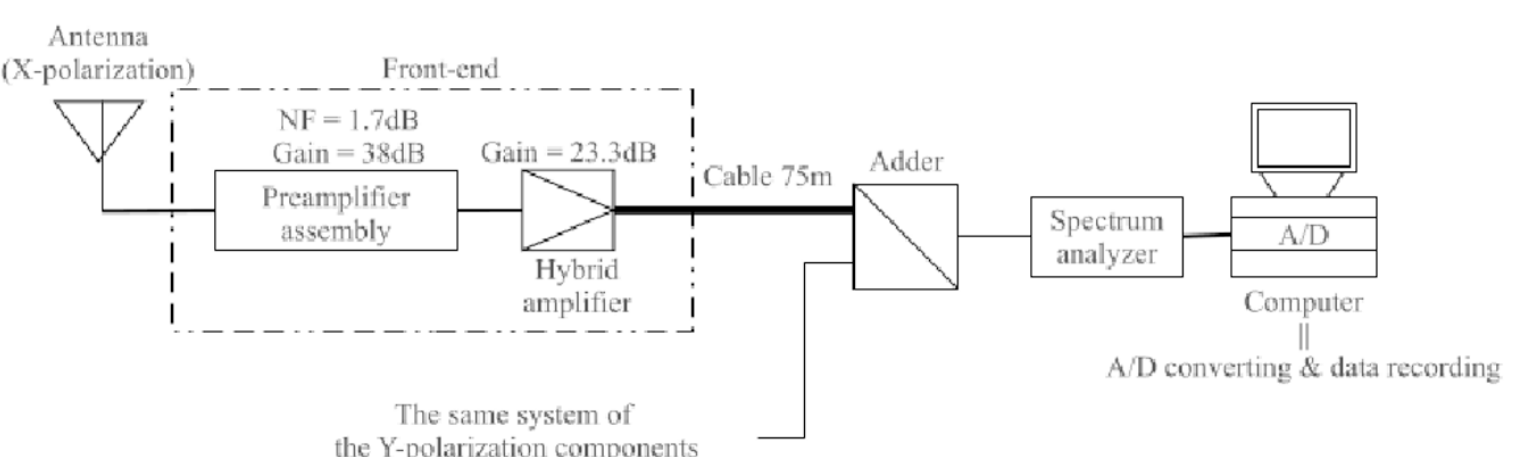

(b)

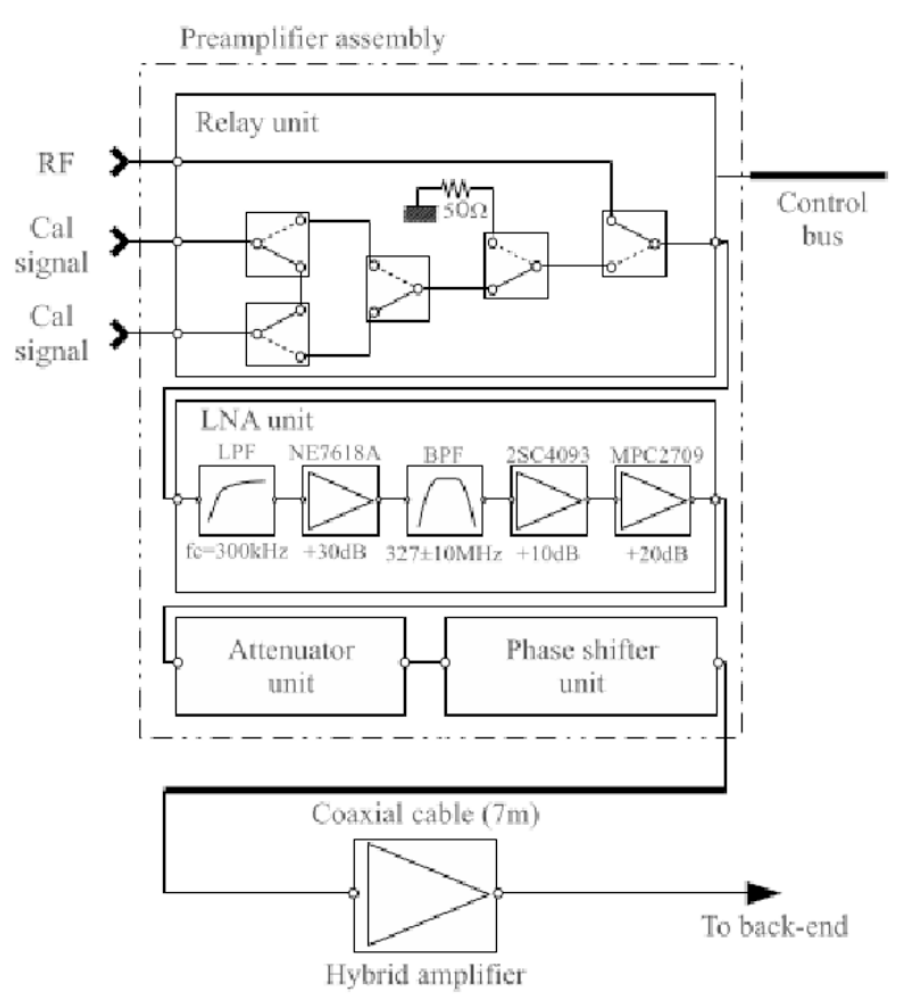

Fig. 1. Schematics of the ZSR. (a) Receiver system. (b) Low noise amplifier (LNA).

(e.g., Magdalenić et al., 2006). However, the time resolution is still not adequate in the meter wavelength range, especially for metric type IV bursts. In addition, the frequency resolution in those observations with high-time resolution has still remained up to $1 \mathrm{MHz}$. Higher-resolution observations and more detailed analyses are required to understand of fine-structured radio bursts, as well as particle and wave dynamics in the solar corona.

In this regard, we established new spectral observations in the range of 315-332 MHz at an extremely high resolution of $100 \mathrm{kHz}$ and $10 \mathrm{~ms}$. The details of the instrumentation are described in Section 2, the statistical characteristics of the observed fine structures are covered in Section 3 and our interpretation is presented in Section 4. Section 5 gives conclusions of the present study.

\section{Instrumentation}

A new instrumentation for the observations of solar radio emission in the meter-wave range, the Zao Solar Radiospectrograph (ZSR), was established in June 2008 at the Zao observatory (latitude: $38^{\circ} 06^{\prime}$, longitude: $140^{\circ} 32^{\prime} \mathrm{E}$, altitude: $685 \mathrm{~m}$ ) of Planetary Plasma and Atmospheric Research Center, Graduate School of Science, Tohoku University, Japan. The observation system is based on system for observation of Jovian synchrotron radiation by Watanabe $e t$ al. (2005). The ZSR system is fed by a $4 \times 2$ stacked $27-$ element cross-Yagi antenna manufactured by Creative Design Corp., covering a frequency range around $327 \mathrm{MHz}$. The antenna effective area is about $20 \mathrm{~m}^{2}$, with a half-power beam width of about $8^{\circ}$. The antenna has a typical altazimuth mount and follows the Sun continuously.

The receiver system consists of an antenna, a front-end equipped with a low-noise amplifier (LNA) unit and a hybrid amplifier, and a back-end with units of a power com- 
Table 1. The ZSR's observation parameters.

\begin{tabular}{lc}
\hline Parameters & ZSR \\
\hline Frequeny range & $315-332 \mathrm{MHz}$ \\
Frequency resolution & $100 \mathrm{kHz}$ \\
Temporal resolution & $10 \mathrm{~ms}$ \\
Antenna & 27-element, 8 -stack Yagi antenna \\
Antenna effective area & $20 \mathrm{~m}^{2}$ \\
Total system gain & $44 \mathrm{~dB}$ \\
Minimum detectable sensitivity & $4.3 \mathrm{SFU}$ \\
\hline
\end{tabular}
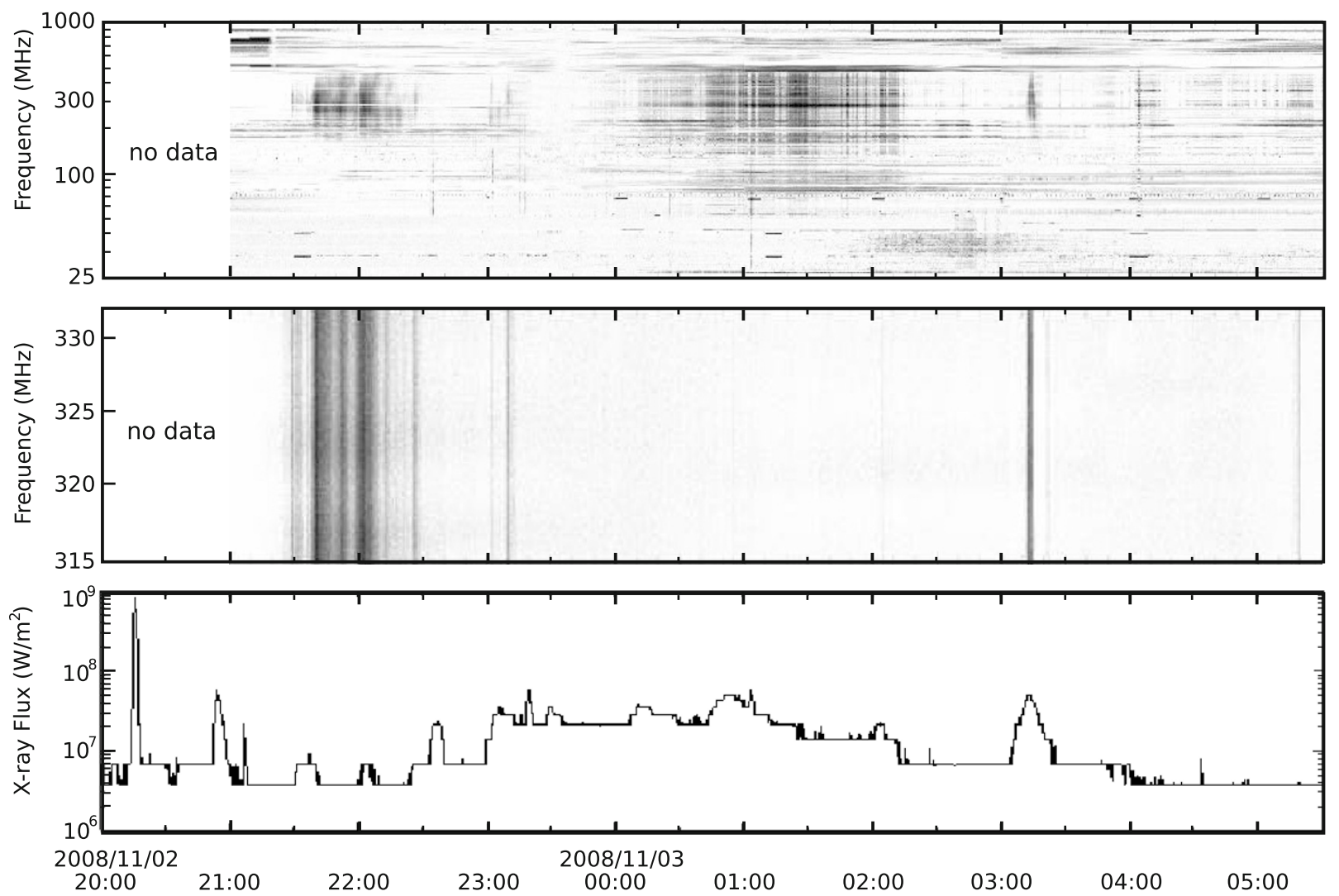

Fig. 2. Overview of the 2 and 3 November 2008 events. Dynamic spectra obtained with (top) Hiraiso Radio Spectrograph (HiRAS) and (middle) the ZSR. (bottom) Light curve of 1-8 § X-ray flux observed by the GOES-10 satellite.

biner, a spectrum analyzer, and a personal computer (PC), as shown in Fig. 1(a). The LNA unit amplifies the input signal with an extremely low level of noise and selects the relevant radio-frequency bandwidth. It has $+40 \mathrm{~dB}$ gain in the frequency range between $317 \mathrm{MHz}$ and $337 \mathrm{MHz}$. The details of the front-end are shown in Fig. 1(b). In this study, both the attenuation level in the programmable attenuator and the phase delay of the programmable phase shifter are set to zero. The hybrid amplifier amplifies the incoming signal using a gain of $+23.3 \mathrm{~dB}$. It is used to compensate the back-end for the power loss caused by the coaxial cable. Finally, the signals are fed into the spectrum analyzer (HP 8558B, manufactured by Hewlett Packard), which receives the signal at frequencies from 315 to $332 \mathrm{MHz}$ at 100 sweeps $\mathrm{s}^{-1}$. The frequency range of the spectrum analyzer is determined with the performance of the antenna and front-end. Information about the amplitude and frequency of the signals are displayed on a Cathode Ray Tube screen, and also transferred as DC voltage signals to the PC's 8-bit
A/D board, which receives 510 samples per sweep. Samples are averaged in thirds, so that the entire spectral range (315-332 MHz) is divided into 170 channels with a resolution bandwidth of $100 \mathrm{kHz}$ each. The converted digital data are stored hourly in binary mode.

In addition to the work by Watanabe et al. (2005), we installed the back-end for spectral observation and the revised antenna control system, and the signal-transmission line was modified to increase the system gain. The ZSR's observation parameters are listed in Table 1. The ZSR has a resolution bandwidth of $100 \mathrm{kHz}$ and a time resolution of $10 \mathrm{~ms}$. The minimum detection sensitivity is $4.3 \mathrm{SFU}$ (1 $\mathrm{SFU}=$ Solar Flux Unit $=10^{-22} \mathrm{~W} \mathrm{~m}^{-2} \mathrm{~Hz}^{-1}$ ).

\section{Observations}

On 2 and 3 November 2008, groups of type IV bursts were observed after a B5.7-class flare occurred in the active region AR 11007. The full view of this event is shown in Fig. 2. The B5.7-class flare started at 20:12 UT on 2 
(a)

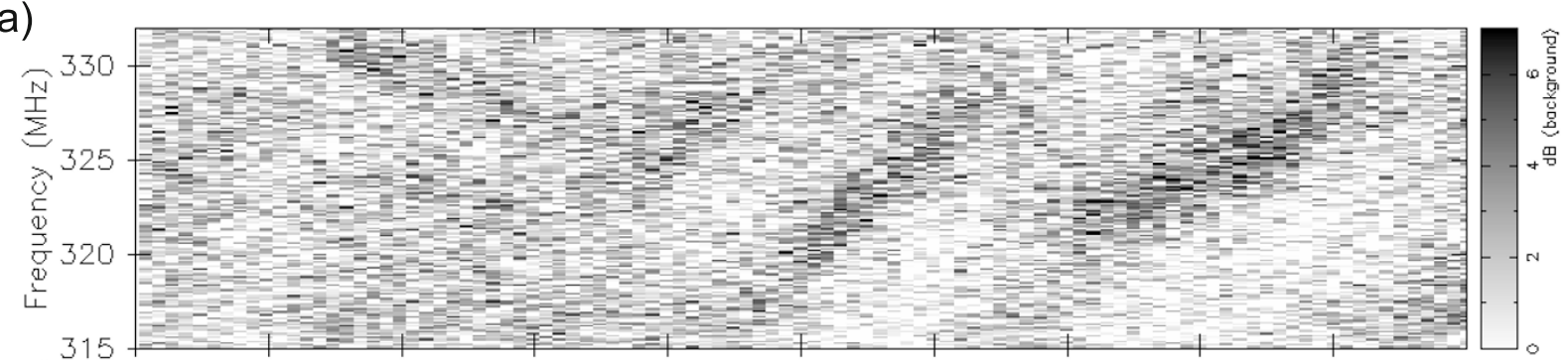

(b)

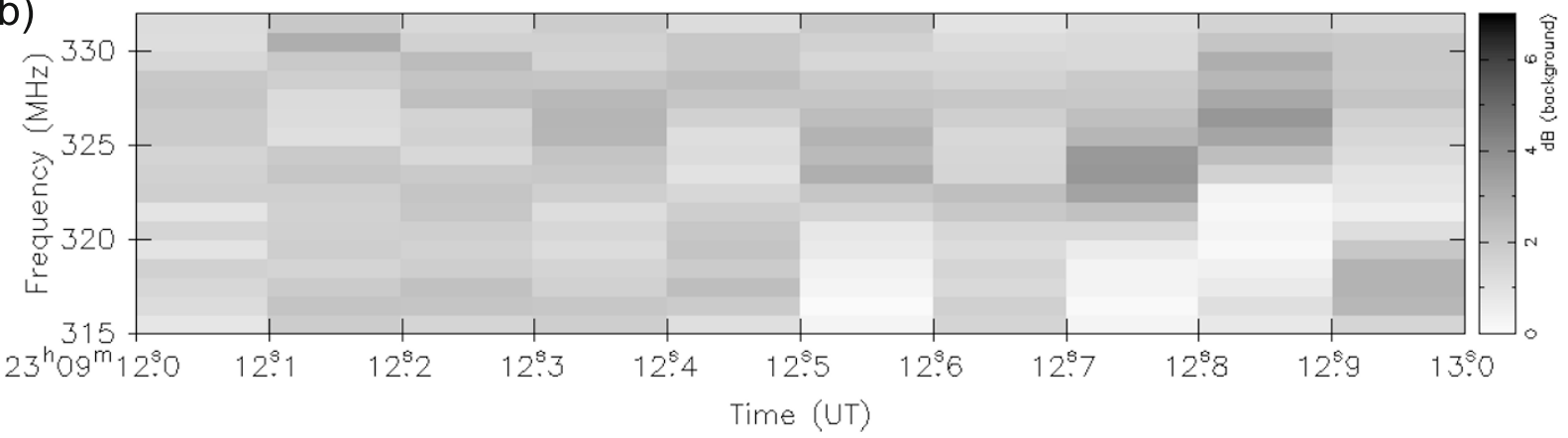

Fig. 3. (a) Dynamic spectrum obtained 23:09:12-13 UT on 2 November 2008. Some narrowband drifting stripes characterized by both positive and negative drift rates are confirmed. (b) Same time span as in panel (a), but with a reduced resolution of $1 \mathrm{MHz}$ and $0.1 \mathrm{~s}$.

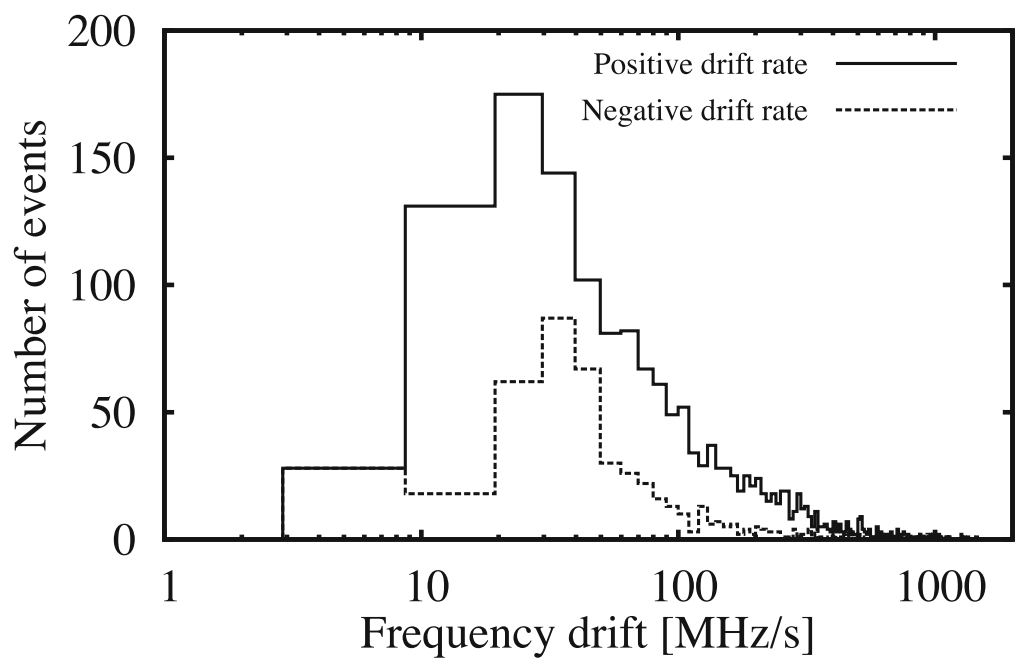

Fig. 4. Histogram of the observed drift rate of fine structures. Solid line: Number distribution of the events with positive frequency drift; dashed line: Equivalent distribution with negative frequency drift.

November 2008, and an A5.0 class flare started at 03:04 UT on 3 November 2008. Both have been confirmed on the basis of an observed GOES-10 light curve. Groups of radio bursts were observed at 21:27-22:30 UT, 23:02-23:14 UT on 2 November, and 03:11-03:15 UT on 3 November. Figure 3(a) shows examples of observed fine structures in type IV bursts. Several drifting stripes characterized by positive frequency drifts are confirmed in this dynamic spectrum. The drift rate of the stripe that starts at 23:09:12.4 UT is $69.2 \mathrm{MHz} \mathrm{s}^{-1}$. The total bandwidth of the stripe is approximately $10 \mathrm{MHz}$. Some stripes also accompany the absorption on the low-frequency side, which is similar to that associated with fiber bursts. For reference, in Fig. 3(b) we plot the dynamic spectrum of this event with a reduced resolution of $1 \mathrm{MHz}$ and $0.1 \mathrm{~s}$ to demonstrate that it is diffi- cult to distinguish the drifting stripes confirmed in Fig. 3(a). This result shows that the resolution generally employed in the previous studies (e.g., Benz et al., 1991) is not sufficient to detect such narrowband drifting stripes and underscores validity of our $100 \mathrm{kHz}$ and $10 \mathrm{~ms}$ high-resolution observations.

Figure 4 shows the number of events with start and end frequencies within the observation's frequency range. Because it is difficult to discuss events characterized by start or end frequencies outside the observed frequency range, we excluded those events. The histogram is binned into $10 \mathrm{MHz} \mathrm{s}^{-1}$ bins of the observed drift rate. The drift rate of each event is derived using least-squares minimization. This result offers the several insights. Fine-structure events with a drift rate of a few tens of $\mathrm{MHz} \mathrm{s}^{-1}$ occur most fre- 
quently, and the number of events with positive drift rates is larger than that of the equivalent events characterized by negative drift rates.

\section{Discussion}

Narrowband emission features with intermediate drift rates observed during this event exhibit some characteristics that are different from typical fiber bursts in type IV bursts.

- About $75 \%$ of events show positive drift rates.

- The distribution of the drift rate has a peak in the range of $20-40 \mathrm{MHz} \mathrm{s}^{-1}$, and only $2.6 \%$ of events have the drift rates of approximately $-9 \mathrm{MHz} \mathrm{s}^{-1}$, a typical value for fiber bursts.

In the physics of solar radio emissions, various emission mechanisms have been studied such as gyro-synchrotron, bremsstrahlung, and plasma emissions. In the observed frequency range, plasma emissions have been mainly considered (e.g., Iwai et al., 2013). In the plasma emission theory, the frequency drift of emission features can be explained by (i) non-thermal electron beams, which are common in type III bursts (e.g., Suzuki and Dulk, 1985), or (ii) whistlermode waves, thought to be the origin of fiber bursts (e.g., Kuijpers, 1975). Therefore, positive drift rates are interpreted as downward motions of the either emission source. In the following sections, we discuss the applicability of these two ideas in the context of the observed level of fine structure from the point of the amplitude of the drift rates.

\subsection{Non-thermal electron beams}

The observed drift rate of emissions can be explained by the radial motion of a wave source in the corona. If radio emissions are generated by wave-wave coupling processes of Langmuir waves excited by non-thermal beam electrons, the emission frequency is determined by the local plasma frequency and thus the drift rate should be related to the speed of beam electrons. Assuming radiation at the fundamental of plasma frequency, the speed $v$ is represented by

$$
v=\frac{\mathrm{d} r}{\mathrm{~d} t} \approx 2 \frac{\mathrm{d} f / \mathrm{d} t}{f} \frac{N_{\mathrm{e}}}{\mathrm{d} N_{\mathrm{e}} / \mathrm{d} r},
$$

where $r$ is the distance from the solar surface $N_{\mathrm{e}}$ is electron density (e.g., Chernov et al., 2008). The energy of an electron beam with a speed corresponding to $40 \mathrm{MHz} \mathrm{s}^{-1}$ is approximately $900 \mathrm{eV}$ in the region with a plasma frequency of $325 \mathrm{MHz}$, applying 10 times the Baumbach-Allen model to the electron-density model in the corona

$$
\begin{aligned}
N_{\mathrm{e}}(r)=10^{9}(2.99 & \left(\frac{r}{r_{\odot}}\right)^{-16}+1.55\left(\frac{r}{r_{\odot}}\right)^{-6} \\
& \left.+0.036\left(\frac{r}{r_{\odot}}\right)^{-1.5}\right) \mathrm{cm}^{-3},
\end{aligned}
$$

where $r_{\odot}$ is the radius of the Sun. Now we will examine whether this velocity of the emission source is non-thermal in the corona (e.g., Aschwanden, 2004).

It is well known that the temperature in the corona after the start of a solar flare often reaches about 10 MK (e.g., Fernandes et al., 2012). Thus, the derived electron energy is at a comparable level to that of the flare related plasma, which seems too small to be a non-thermal electron beam generating radio emission. Therefore, we conclude that fine-structure events with a drift rate of a few tens of $\mathrm{MHz}$ $\mathrm{s}^{-1}$ are not caused by non-thermal electron beams. It should be noted that the magnetic field lines along which the emission sources move are assumed to be completely perpendicular to the solar surface. If the magnetic lines are oblique to the radial direction, the speed of the motion of the wave source corresponding to the observed drift rate can be larger than the estimated value. We do not rule out non-thermal electron beams moving along oblique magnetic field lines as a possible wave source of the event with a drift rate of a few tens of $\mathrm{MHz} \mathrm{s}^{-1}$

On the other hand, events characterized by the higher drift rates, especially above $100 \mathrm{MHz} \mathrm{s}^{-1}$ which corresponds to about $7.6 \mathrm{keV}$ of the energy of electrons, seem to have their origin in such an electron beam because they can have the higher speed and be an non-thermal electron beam.

\subsection{Whistler-mode waves}

Equation (1) represents the relation between the speed of the motion of an emission source and the frequency drift rate of radio waves. Here we consider a wave-wave coupling process between Langmuir waves and whistler-mode waves in the corona. In this coupling process, radio emissions are generated at the frequency close to the plasma frequency. In a case that the emission source where this coupling process occurs moves radially in coronal plasma, where the local plasma frequency varies radially, the emission frequency varies in time. Assuming that the observed radio bursts are related to whistler-mode waves, the speed of the motion of the emission source is equal to the group velocity of whistler-mode wave packets. Therefore, in the whistler-mode wave framework, $v$ in Eq. (1) can be replaced by the group velocity of whistler-mode waves $v_{\mathrm{g}}$. In the quasi-longitudinal case, $v_{\mathrm{g}}$ is represented by

$$
v_{\mathrm{g}}=2 c \frac{\omega_{\mathrm{ce}}}{\omega_{\mathrm{p}}} \sqrt{x(1-x)^{3}}
$$

where $c$ is the speed of light, $\omega_{\mathrm{ce}}$ is electron cyclotron frequency, $\omega_{\mathrm{p}}$ is plasma frequency, $x=\omega / \omega_{\text {ce }}$ is the ratio of emission frequency and electron cyclotron frequency (Kuijpers, 1975). The calculation results of the speed at the region where the plasma frequency are equal to $325 \mathrm{MHz}$ and $\omega_{\mathrm{p}} / \omega_{\mathrm{ce}}=10$ is shown in Fig. 5 and it shows that the maximum velocity at the observed region is about $0.065 \mathrm{c}$. By using Eqs. (1) and (2), the frequency drift rate corresponding to speed of $0.065 c$ is derived as $39.04 \mathrm{MHz} \mathrm{s}^{-1}$. This is approximately the same as the observed drift rates.

In addition to whistler-mode waves, Alfvén velocity has also been proposed as the speed of the wave source motion for the mechanism of frequency drift emissions. Here we evaluate Alfvén velocity for the agent generating fiber bursts (e.g., Treumann et al., 1990). The Alfvén velocity $v_{\mathrm{A}}$ is represented by

$$
v_{\mathrm{A}}=\frac{B}{\sqrt{\mu_{0} n_{\mathrm{i}} m_{\mathrm{i}}}},
$$

where $B$ is the strength of magnetic field, $\mu_{0}$ is the magnetic permeability, $n_{\mathrm{i}}$ and $m_{\mathrm{i}}$ are the number density and mass of 

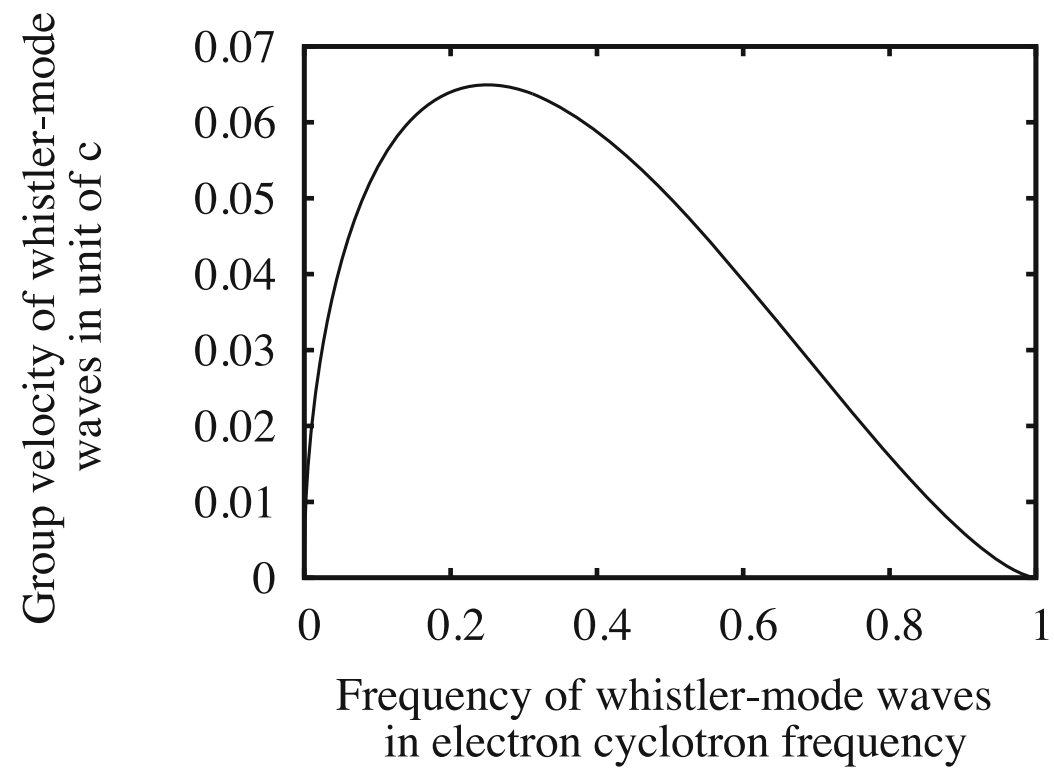

Fig. 5. The group velocity of whistler-mode waves in the coronal region with a plasma frequency of $325 \mathrm{MHz}$.

ions. We use the electron density in the observed coronal region for $n_{\mathrm{i}}=1.30 \times 10^{9} \mathrm{~cm}^{-3}$ and the proton mass for $m_{\mathrm{i}}$. If the $40 \mathrm{MHz} \mathrm{s}^{-1}$ drift rate is assumed to be explained by Alfvén velocity, the magnetic field strength should be approximately $300 \mathrm{G}$ in the observed coronal region. The magnetic field strength is estimated to be $12 \mathrm{G}$ by the model of Dulk and McLean (1978) in the region of the plasma frequency of $325 \mathrm{MHz}$. Since the derived magnetic field is too large for the plausible source region at $0.12 R_{\mathrm{S}}$ from the surface of the sun, it is difficult to explain the observed drift speed as Alfvén velocity.

Next, we study the coupling condition of the plasma waves for radio emission through the interaction of a Langmuir and a whistler-mode waves to an L-O mode wave (hereafter we denote this process as $L+w \rightarrow T$ ). In a cold plasma, the dispersion relation of waves is described by the Appleton-Hartree equation,

$$
n_{ \pm}^{2}=1-\frac{X(1-X)}{1-X-\frac{Y^{2}}{2} \sin ^{2} \theta \pm \sqrt{\frac{Y^{4}}{4} \sin ^{4} \theta+4\left(1-X^{2}\right) Y^{2} \cos ^{2} \theta}},
$$

where $X=\omega_{\mathrm{p}}^{2} / \omega^{2}, Y=\omega_{\mathrm{ce}} / \omega, n=c k / \omega$, and $\theta$ is the wave-propagation angle with respect to the background magnetic field.

Figure 6 shows the dispersion relation calculated for $\omega_{\mathrm{p}} / \omega_{\mathrm{ce}}=10$ and $f_{\mathrm{p}}=\omega_{\mathrm{p}} / 2 \pi=325 \mathrm{MHz}$, which is derived from 10 times the Baumbach-Allen model for the coronal electron density and the Dulk and Mclean model for the coronal magnetic field strength (Dulk and McLean, 1978). With this result and the conservation law of

$$
\omega_{\mathrm{w}}+\omega_{\mathrm{L}}=\omega_{\mathrm{T}}
$$

and

$$
\vec{k}_{\mathrm{W}}+\vec{k}_{\mathrm{L}} \rightarrow \vec{k}_{\mathrm{T}},
$$

where $k$ is the wave number, we can find the wave number fulfill the coupling conditions of Eqs. (6) and (7) for the frequency of $\omega_{\mathrm{w}}=0.25 \omega_{\mathrm{ce}}$ based on the discussion on Fig. 5 and $\omega_{\mathrm{L}}=\omega_{\mathrm{p}}$. The absolute values of the wave number become $k_{\mathrm{L}}=6.28 \mathrm{~m}^{-1}$ and $k_{\mathrm{T}}=2.35 \mathrm{~m}^{-1}$, where a propagation angle of the $\mathrm{L}-\mathrm{O}$ wave is $0^{\circ}$. These solutions are indicated by arrows onto branches in Fig. 6 . This shows that the three-wave coupling of $L+w \rightarrow T$ can take place with whistler-mode waves of the frequency of $\omega_{\mathrm{w}}=0.25$ $\omega_{\text {ce }}$.

It is demonstrated that whistler-mode waves with a group velocity corresponding to the observed drift rate of order 40 $\mathrm{MHz} \mathrm{s}^{-1}$, which is not typical for the fiber bursts, can exist and that the observed drift emission can be generated by whistler-mode waves. In this study, the frequency of whistler-mode waves of $0.25 \omega_{\text {ce }}$ at which the group velocity is maximal is taken account, whereas the frequency of whistler-mode waves was assumed approximately $0.1 \omega_{\text {ce }}$ in previous studies of fiber bursts (e.g., Chernov et al., 2008). This is the main reason why the fine structures with a drift rate of a few tens of $\mathrm{MHz} \mathrm{s}^{-1}$ is explained by the same mechanism of typical fiber bursts which have approximate $-9 \mathrm{MHz} \mathrm{s}^{-1}$ in the observed frequency range.

In the present study, we assumed that whistler-mode waves are excited by energetic electrons generated in the associated flare. Here we consider the plasma environment in the coronal region. In the observed coronal region where the plasma frequency is around $325 \mathrm{MHz}$, the ratio of the plasma frequency to the cyclotron frequency, $\omega_{\mathrm{p}} / \omega_{\mathrm{ce}}$ is estimated to be 10 . This condition is similar to the plasma environment in the terrestrial magnetosphere, where whistlermode waves are generated under the presence of energetic electrons of a few to tens of $\mathrm{keV}$ having anisotropic velocity distribution (e.g., Kennel and Petschek, 1966). Since $\mathrm{keV}$ electrons should be ubiquitous in the coronal region after flare, we can expect the generation of whistler-mode waves at the frequency range around $0.25 \omega_{\mathrm{ce}}$ assumed in the present study.

Events that exhibit faster drift rates than about $100 \mathrm{MHz}$ $\mathrm{s}^{-1}$, on the other hand, cannot be explained by the whistlermode waves. They are assumed to be generated by nonthermal electron beams as well as type III bursts. The char- 


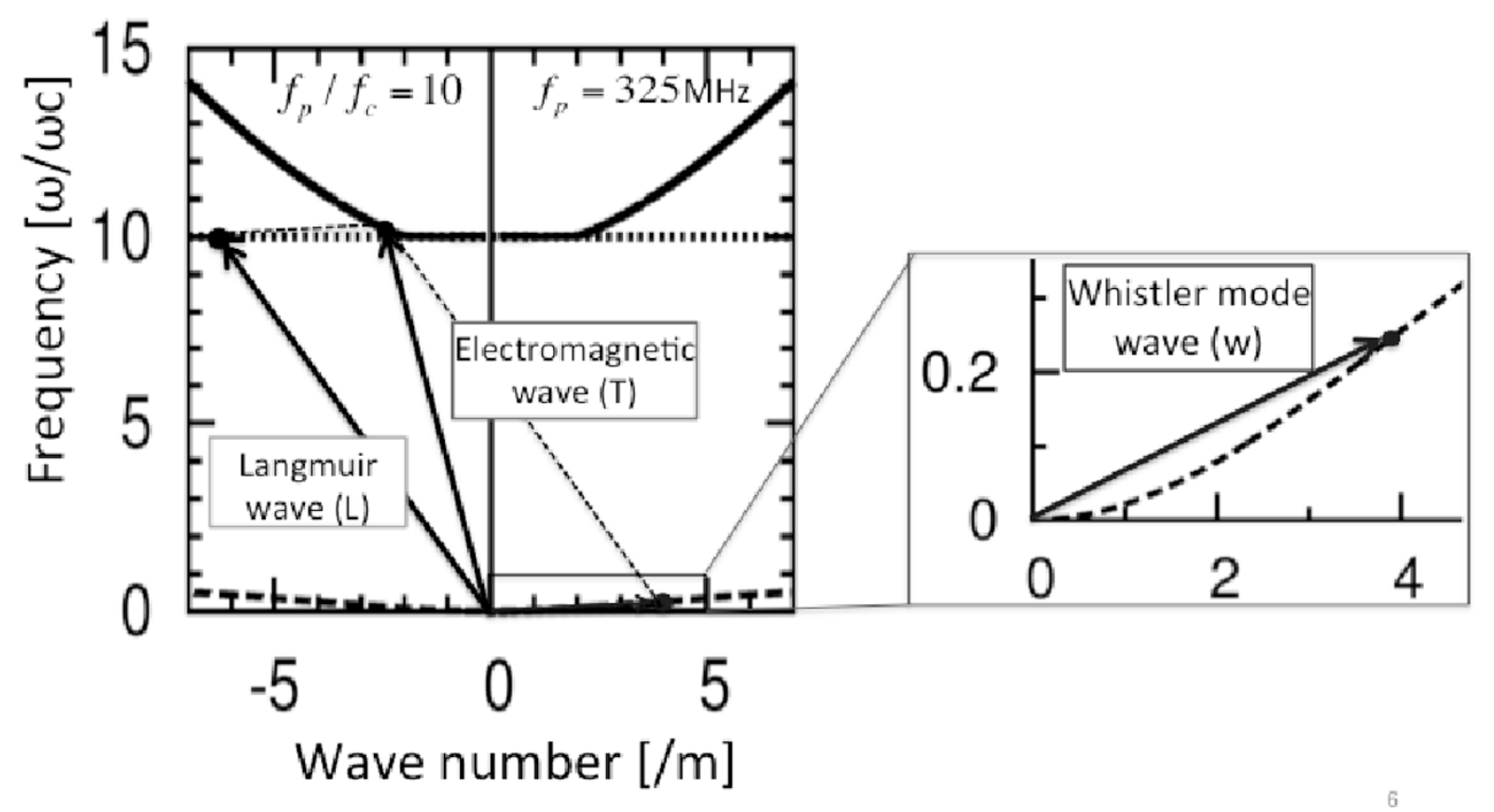

Fig. 6. Calculated wave-dispersion relation based on the Appleton-Hartree equation for $\omega_{\mathrm{p}} / \omega_{\mathrm{ce}}=10, f_{\mathrm{p}}=\omega_{\mathrm{p}} / 2 \pi=325 \mathrm{MHz}$ and $\theta=0{ }^{\circ}$. The solid line shows L-O mode wave, the dotted line does Langmuir wave, and the dashed line does whistler-mode wave. The arrows on the graph indicate the solution of the non-linear three-wave interaction, $L+w \rightarrow T$, for whistler-mode waves of $0.25 \omega_{\text {cee }}$.

acteristic drift rate that divides the two generation mechanisms discussed above is not clear from the histogram in Fig. 4.

\section{Conclusion}

To investigate fine-structure details of solar type IV bursts, we established a new radio observation system at the Zao observatory of Tohoku University. Narrowband fine structures that are difficult to detect based on observations with typical spectral resolutions were observed in type IV bursts on 2 and 3 November 2008. Statistical analysis of the drift rate shows that they are different from ordinary fiber bursts in both sense and magnitude. First, the observed drift rates exhibit both positive and negative rates, whereas ordinary fiber bursts usually have negative drift rates. Second, the absolute values of the observed drift rates are tens of $\mathrm{MHz} \mathrm{s}^{-1}$, while the typical drift rate of fiber bursts at $325 \mathrm{MHz}$ is approximately $-9 \mathrm{MHz} \mathrm{s}^{-1}$ (e.g., Benz and Mann, 1998). In addition, the all analyzed fine structures have narrow emission bands of less than $17 \mathrm{MHz}$. The observed narrowband emissions features with drift rates of a few tens of $\mathrm{MHz} \mathrm{s}^{-1}$ at $325 \mathrm{MHz}$ can be interpreted by the generation process of fiber bursts (Kuijpers, 1975); i.e., the emission features are thought to be caused by whistlermode waves propagating in the corona. These observed narrowband events are difficult to detect with the traditional resolution of $1 \mathrm{MHz}$ and $0.1 \mathrm{~s}$. These results imply that the higher resolution used in the present study is necessary to investigate fine structures accompanying type IV bursts.

Based on the observations using ZSR, only one event was detected, which consists of the radio burst with narrowband fine structure. The number of events is too small to discuss their occurrence rate. In addition, the ZSR's frequency range is too narrow to survey their association with other radio bursts in different frequency range and the variance of the coronal loop structures and the distribution of energetic electrons obtained through imaging observations such as with Hinode, STEREO, SDO, and the Nobeyama Radio Heliograph. This problem can be overcome with new wide-band and high-resolution observations of solar radio bursts, such as obtained with IPRT/AMATERAS (Iwai et al., 2012).

Acknowledgments. The ZSR instrumentation is supported by the Planetary Plasma and Atmosphere Research Center, Graduate School of Science, Tohoku University. The author wishes to thank Drs. Maki Akioka and Tetsuro Kondou who provided radio spectra observed at HiRAS. This work was carried out as part of a joint research program with the Solar-Terrestrial Environment Laboratory at Nagoya University. This work was also supported by the Global COE (Center of Excellence) program, 'Global Education and Research Center for Earth and Planetary Dynamics', at Tohoku University (Principal Investigator: Prof. Eiji Ohtani).

\section{References}

Aschwanden, M. J., Physics of the Solar Corona. An Introduction, 842 pp, Praxis Publishing Ltd, Chichester (UK), 2004.

Aurass, H., G. P. Chernov, M. Karlický, J. Kurths, and G. Mann, On a sequence of remarkable fine structures in the type IV burst of 24 April, 1985, Sol. Phys., 112, 347-357, 1987.

Aurass, H., G. Rausche, G. Mann, and A. Hofmann, Fiber bursts as 3D coronal magnetic field probe in postflare loops, Astron. Astrophys., 435, 1137-1148, 2005.

Benz, A. O. and G. Mann, Intermediate drift bursts and the coronal magnetic field, Astron. Astrophys., 333, 1034-1042, 1998.

Benz, A. O., M. Güdel, H. Isliker, S. Miszkowicz, and W. Stehling, A broadband spectrometer for decimetric and microwave radio bursts: first results, Sol. Phys., 133, 385-393, 1991.

Bernold, T. E. X. and R. A. Treumann, The fiber fine structure during solar type IV radio bursts: observations and theory of radiation in presence of localized whistler turblence, Astrophys. J., 264, 677-688, 1983. 
Chernov, G. P., Solar radio bursts with drifting stripes in emission and absorption, Space Sci. Rev., 127, 195-326, 2006.

Chernov, G. P., L. V. Yasnov, Y. Yan, and Q. Fu, On the zebra structure in the frequency range near $3 \mathrm{GHz}$, Chin. J. Astron. Astrophys., 1, 525-536, 2001.

Chernov, G. P., Y. Yan, Q. Fu, C. Tan, and S. Wang, Unusual zebra patterns in the decimeter wave band, Sol. Phys., 250, 115-131, 2008.

Dąbrowski, B. P., P. Rudawy, and M. Karlický, Millisecond radio spikes in the decimetric band, Sol. Phys., 273, 377-392, 2011.

Dulk, G. A. and D. J. McLean, Coronal magnetic fields, Sol. Phys., 57, 279-295, 1978.

Elgarøy, Ø., Frequency drift and fine structure of $200 \mathrm{Mc} / \mathrm{s}$ solar bursts, in URSI Symp. 1: Paris Symp. on Radio Astron., edited by R. N. Barcewell, 248 pp, Stanford Univ. Press, Stanford, CA, 1959.

Fernandes, F. C. R., V. Krishan, M. C. Andrade, J. R. Cecatto, D. C. Freitas, and H. S. Sawant, High resolution studies of intermediate drift bursts, Adv. Space Res., 32, 2545-2550, 2003.

Fernandes, F. C. R., J. A. S. S. Dutra, R. D. Cunha da Silva, and H. S. Sawant, Flaring loop parameters estimated from solar decimeter type U-like and type J-like fine structures, Adv. Space Res., 49, 1607-1614, 2012.

Fu, Q., Z. Qin, H. Ji, and L. Pei, A broadband spectrometer for decimeter and microwave radio bursts, Sol. Phys., 160, 97-103, 1995.

Iwai, K., F. Tsuchiya, A. Morioka, and H. Misawa, IPRT/AMATERAS: A new metric spectrum observation system for solar radio bursts, Sol. Phys., 277, 447-457, 2012

Iwai, K., S. Masuda, Y. Miyoshi, F. Tsuchiya, A. Morioka, and H. Misawa, Peak flux distributions of solar radio type-I bursts from highly resolved spectral observations, Astrophys. J. Lett., 768, L2, 2013.

Jiřička, K. and M. Karlický, Narrowband pulsating decimetric structure observed by the New Ondřejov solar radio spectrograph, Sol. Phys., 253, 95-101, 2008.

Karlický, M., H. Mèszárosová, and P. Jelínek, Radio fiber bursts and fast magnetoacoustic wave trains, Astron. Astrophys., 550, A1, 2013.

Kennel, C. F. and H. E. Petschek, Limit on stably trapped particle fluxes, J. Geophys. Res., 71, 1-28, 1966.

Kondo, T., T. Isobe, S. Igi, S. Watari, and M. Tokumaru, The Hiraiso Radio Spectrograph (HiRAS) for monitoring solar radio bursts, J. Commun. Res. Lab., 42, 111-119, 1995.
Kuijpers, J., Generation of intermediate drift bursts in solar type IV radio continua through coupling of whstlers and Langmuir waves, Sol. Phys. 44, 173-193, 1975.

Kuznetsov, A. A., Generation of intermediate drift bursts by magnetohydrodynamic waves in the solar corona, Sol. Phys., 237, 153-171, 2006.

Magdalenić, J., B. Vršnak, P. Zlobec, A. Hillaris, and M. Messerotti, Classification and properties of supershort solar radio bursts, Astrophys. J., 642, L77, 2006

Prestage, N. P., R. G. Luckhurst, B. R. Paterson, C. S. Bevins, and C. G. Yuile, A new radiospectrograph at culgoora, Sol. Phys., 150, 393-396, 1994.

Sawant, H. S., K. R. Subramanian, C. Faria, F. C. R. Fernandes, J. H. A. Sobral, J. R. Cecatto, R. R. Rosa, V. O. Vats, J. A. C. F. Neri, E. M. B. Alonso, F. P. V. Mesquita, V. A. Portezani, and A. R. F. Martinon, Brazilian solar spectroscope (BSS), Sol. Phys., 200, 167-176, 2001.

Slottje, C., Peculiar absorption and emission microstructures in the type IV solar radio outburst of March 2, 1970, Sol. Phys., 25, 210-231, 1972.

Suzuki, S. and G. A. Dulk, Bursts of type III and type V, in Solar Radiophysics: Studies of Emission from the Sun at Metre Wavelengths, edited by D. J. Mclean and N. R. Labrum, Canbridge Univ. Press, Cambridge \& New York, 1985

Thompson, A. R. and A. Maxwell, Spectral observations of solar radio bursts. III. Continuum bursts, Astrophys. J., 136, 546-573, 1962.

Treumann, R. A., M. Güdel, and A. O. Benz, Alfvén wave solitons and solar intermediate drift bursts, Astron. Astrophys., 236, 242-249, 1990.

Watanabe, T., H. Misawa, F. Tsuchiya, Y. Miyoshi, T. Abe, and A. Morioka, Development of the observation system for the Jovian synchrotron radiation using an aperture synthesis array, Tohoku Geophys. J., 37, 1-90, 2005

Wild, J. P., S. F. Smerd, and A. A. Weiss, Solar bursts, Ann. Rev. Astron. Astrophys., 1, 291-366, 1963.

Young, C. W., C. L. Spencer, G. E. Moreton, and J. A. Roberts, A preliminary study of the dynamic spectra of solar radio bursts in the frequency range 500-950 Mc/s, Astrophys. J., 133, 243-264, 1961.

Y. Nishimura (e-mail: yukio@stpp.gp.tohoku.ac.jp), T. Ono, F. Tsuchiya, H. Misawa, A. Kumamoto, Y. Katoh, S. Masuda, and Y. Miyoshi 